COMMUNICATIONS IN

ANALYSIS AND GEOMETRY

Volume 13, Number 5, 903-928, 2005

\title{
Surface bundles versus Heegaard splittings
}

\author{
David Bachman and Saul Schleimer ${ }^{1}$
}

This paper studies Heegaard splittings of surface bundles via the curve complex of the fibre. The translation distance of the monodromy is the smallest distance it moves any vertex of the curve complex. We prove that the translation distance is bounded above in terms of the genus of any strongly irreducible Heegaard splitting. As a consequence, if a splitting surface has small genus compared to the translation distance of the monodromy, then the splitting is standard.

\section{Introduction.}

The purpose of this paper is to show a direct relationship between the action of a surface automorphism on the curve complex and the Heegaard splittings of the associated surface bundle.

We begin by restricting attention to automorphisms of closed orientable surfaces with genus at least two. Let $\varphi: F \rightarrow F$ be such an automorphism. The translation distance, $d_{\mathcal{C}}(\varphi)$, is the shortest distance $\varphi$ moves any vertex in the curve complex. (We defer precise definitions to Section 2.) As a bit of notation, let $M(\varphi)$ denote the mapping torus of $\varphi$. The following theorem, alluded to in [21], gives a link between translation distance and essential surfaces in $M(\varphi)$.

Theorem 3.1. If $G \subset M(\varphi)$ is a connected, orientable, incompressible surface, then either $G$ is isotopic to a fibre, $G$ is homeomorphic to a torus, or $d_{\mathcal{C}}(\varphi) \leq-\chi(G)$.

An underlying theme in the study of Heegaard splittings is that, in many instances, strongly irreducible splitting surfaces may take the place of incompressible surfaces. We prove:

Theorem 6.1. If $H$ is a strongly irreducible Heegaard splitting of $M(\varphi)$, then

$$
d_{\mathcal{C}}(\varphi) \leq-\chi(H)
$$

\footnotetext{
${ }^{1}$ This work is in the public domain
} 
This result gives new information about Heegaard splittings of hyperbolic three-manifolds. Previous work, by Moriah and Rubinstein [14], discussed the low genus splittings of negatively-curved manifolds with very short geodesics. Restricting attention to surface bundles and applying Theorems 6.1 and 3.1 gives:

Corollary 3.2. Any Heegaard splitting $H$ of the mapping torus $M(\varphi)$ with $-\chi(H)<d_{\mathcal{C}}(\varphi)$ is a stabilization of the standard splitting.

This improves results due to Rubinstein [16] and Lackenby [11] (see Remark 3.5).

The two theorems indicate an interesting connection between the combinatorics of the curve complex and the topology of three-manifolds. This accords with other work. Most significantly, Minsky et al. [3] have used the curve complex to prove the Ending Lamination Conjecture. A major step is using a path in the curve complex to give a model of the geometry of a hyperbolic three-manifold.

Another example of this connection is found in [7] and [8]. These papers study surface bundles where the fibre is a once punctured torus. Here, the Farey graph takes the place of the curve complex. An analysis of $\varphi$-invariant lines in the Farey graph allows a complete classification of incompressible surfaces in such bundles.

As we shall see in the proofs of Theorems 3.1 and 6.1, essential surfaces and strongly irreducible splittings in the mapping torus $M(\varphi)$ yield $\varphi$-invariant lines in the curve complex of the fibre. It is intriguing to speculate upon axioms for such lines which would, perhaps, lead to classification results for essential surfaces or strongly irreducible splittings. This would be a significant step in the over-all goal of understanding the topology of surfaces bundles of the circle.

At the heart of our proof of Theorem 6.1 lies the idea of a "graphic", due to Rubinstein and Scharlemann [17]. The graphic is obtained, as in Cooper and Scharlemann's paper [6], by comparing the bundle structure with a given height function and applying Cerf theory. As in their work, our situation requires a delicate analysis of behavior at the vertices of the graphic.

The rest of the paper is organized as follows: basic definitions regarding Heegaard splittings, surface bundles, and the curve complex are found in Section 2. With this background, we restate the main theorem and corollaries in Section 3. Of main importance is the nature of simple closed curve intersections between a fibre of the bundle and the Heegaard splitting un- 
der discussion. This is covered in Section 4, in addition to a preliminary sketch of the proof of Theorem 6.1. The Rubinstein-Scharlemann graphic is discussed in Section 5. Concluding the paper, Section 6 proves Theorem 6.1 and poses a few open questions. We also discuss the possibility of strengthening the inequality given in the main theorem to a more refined "moduli space." For example, translation distance in the pants complex or in Teichmüller space does not work.

\section{Background material.}

This section presents the definitions used in this paper. A more complete reference for the curve complex may be found in [13] while the paper [19] is an excellent survey on Heegaard splittings.

\subsection{The curve complex.}

Fix a closed connected orientable surface $F$ with genus $g(F) \geq 2$. If $\alpha \subset F$ is an essential simple closed curve, then let $[\alpha]$ be the isotopy class of $\alpha$.

Definition 2.1. The set $\left\{\left[\alpha_{0}\right], \ldots,\left[\alpha_{k}\right]\right\}$ determines a $k$-simplex if for all $i \neq j$ the isotopy classes $\left[\alpha_{i}\right],\left[\alpha_{j}\right]$ are distinct and there are $\alpha_{i}^{\prime} \in\left[\alpha_{i}\right]$, $\alpha_{j}^{\prime} \in\left[\alpha_{j}\right]$ with $\alpha_{i}^{\prime} \cap \alpha_{j}^{\prime}=\emptyset$.

Definition 2.2. The curve complex of $F$ is the simplicial complex $\mathcal{C}(F)$ given by the union of all simplices, as above.

We will restrict our attention to the zero and one-skeleta, $\mathcal{C}^{0}(F) \subset \mathcal{C}^{1}(F)$. Giving each edge length one, the graph $\mathcal{C}^{1}(F)$ becomes a metric space. Let $d_{\mathcal{C}}(\alpha, \beta)$ be the distance between the vertices $[\alpha],[\beta] \in \mathcal{C}^{0}(F)$. When it can cause no confusion, we will not distinguish between an essential simple closed curve and its isotopy class.

Definition 2.3. Suppose that $\varphi$ is a homeomorphism of $F$. The translation distance of $\varphi$ is

$$
d_{\mathcal{C}}(\varphi)=\min \left\{d_{\mathcal{C}}(\alpha, \varphi(\alpha)) \mid \alpha \in \mathcal{C}^{0}(F)\right\} .
$$

\subsection{Heegaard splittings.}

Recall that a compression-body is a three-manifold $V$ obtained as follows: choose a closed connected orientable surface $H$ which is not a two-sphere. 
Form the product $H \times I$. Attach two-handles to the boundary component $H \times\{0\}$. Glue three-handles to all remaining boundary components which are two-spheres to finally obtain $V$. Denote $H \times\{1\}$ in $V$ by $\partial_{+} V$. If the resulting compression-body has $\partial V=\partial_{+} V$, then it is a handlebody.

Let $M$ be a compact connected orientable three-manifold.

Definition 2.4. A surface $H \subset M$ is a Heegaard splitting of $M$ if $H$ cuts $M$ into a pair of compression-bodies $V, W$ and $\partial_{+} V=H=\partial_{+} W$.

Definition 2.5. A properly embedded disk $D$ inside a compression-body $V$ is essential if $\partial D \subset \partial_{+} V$ is essential.

Definition 2.6. A Heegaard splitting $H \subset M$ is stabilized if there are a pair of essential disks $D \subset V$ and $E \subset W$ where $\partial D$ and $\partial E$ intersect in a single point.

Compressing $H$ along $D$ gives a surface $H^{\prime}$; it is an easy exercise to show that $H^{\prime}$ is again a Heegaard splitting of $M$. In this situation, we call $H$ a stabilization of $H^{\prime}$. We now have a more important notion, introduced by Casson and Gordon [5]:

Definition 2.7. A Heegaard splitting $H \subset M$ is strongly irreducible if all pairs of essential disks $D \subset V$ and $E \subset W$ satisfy $\partial D \cap \partial E \neq \emptyset$.

If the splitting $H$ is not strongly irreducible, then $H$ is weakly reducible. Scharlemann's [18] "no nesting" lemma shows the strength of Casson and Gordon's definition:

Lemma 2.8. Suppose $H \subset M$ is a strongly irreducible splitting and $D \subset M$ is an embedded disk with $\partial D \subset H$ and with interior $(D)$ transverse to $H$. Then there is a disk $D^{\prime}$ properly embedded in $V$ or $W$ with $\partial D^{\prime}=\partial D$.

We take the following bit of terminology almost directly from [20]. Let $M=F \times I$ where $F$ is a closed orientable surface. Let $\alpha=\{\mathrm{pt}\} \times \mathrm{I}$ be a properly embedded arc. Take $N$ to be a closed regular neighborhood of $\partial M \cup \alpha$ in $M$. Let $H=\partial N \backslash \partial M$. Then $H$ is the standard type 2 splitting of $M$. The standard type 1 splitting is isotopic to the surface $F \times\{1 / 2\}$.

Scharlemann and Thompson then prove:

Theorem 2.9. Every Heegaard splitting of $M=F \times I$ is a stabilization of the standard type 1 or 2 splitting.

Note that it is common to refer to stabilizations of a standard splitting as being standard themselves. 


\subsection{Surface bundles.}

Fix $F$, a closed, orientable surface with genus $g(F) \geq 2$. Let $\varphi: F \rightarrow F$ be a surface diffeomorphism which preserves orientation.

Definition 2.10. The surface bundle with monodromy $\varphi$ is the manifold

$$
M(\varphi)=(F \times[0,2 \pi]) /\{(x, 2 \pi) \equiv(\varphi(x), 0)\} .
$$

Let $F(\theta)$ be the image of $F \times \theta$. These surfaces are fibres of the bundle $M(\varphi)$. There is a natural smooth map $\pi_{F}: M(\varphi) \rightarrow S^{1}$ defined by $\pi_{F}(x)=$ $\theta$ whenever $x \in F(\theta)$. The map $\pi_{F}$ realizes $M(\varphi)$ as a surface bundle over the circle.

We now define the standard Heegaard splitting of the surface bundle $M(\varphi)$. Pick $x, y \in F$ such that $x \neq y$ and $\varphi(y) \neq x$. Fix $A$ and $B$ disjoint closures of regular neighborhoods of $x \times[0, \pi]$ and $y \times[\pi, 2 \pi]$ respectively. Set

$$
V=\overline{(F \times[\pi, 2 \pi] \backslash B) \cup A}
$$

and

$$
W=\overline{(F \times[0, \pi] \backslash A) \cup B} .
$$

Then $H=\partial V=\partial W$ is the standard Heegaard splitting of $M(\varphi)$. Note that the genus of the standard splitting is $2 g(F)+1$. Finally, the standard splitting is always weakly reducible because the tubes $A \cap H$ and $B \cap H$ admit disjoint compressing disks.

\section{Main theorem and corollaries.}

Let $F$ be a closed orientable surface with genus $g(F) \geq 2$. Let $\varphi: F \rightarrow F$ be an orientation-preserving diffeomorphism. The surface bundle $M(\varphi)$ is irreducible and has minimal Heegaard genus two or larger. Here is a precise statement of our main theorem:

Theorem 6.1. If $H \subset M(\varphi)$ is a strongly irreducible Heegaard splitting, then the translation distance of $\varphi$ is at most the negative Euler characteristic of $H$. That is,

$$
d_{\mathcal{C}}(\varphi) \leq-\chi(H)
$$

This theorem is a deeper version of the following (claimed in [21]): 
Theorem 3.1. If $G \subset M(\varphi)$ is a connected, orientable, incompressible surface, then either

- $G$ is isotopic to a fibre or

- $G$ is a torus and $d_{\mathcal{C}}(\varphi) \leq 1$ or

- $d_{\mathcal{C}}(\varphi) \leq-\chi(G)$.

For completeness, we include a proof.

Proof of Theorem 3.1. Suppose that $G$ is not isotopic to a fibre of the surface bundle. If $G$ is a torus, then the map $\varphi$ is reducible. It follows that $d_{\mathcal{C}}(\varphi) \leq 1$. Assume, therefore, that $G$ is not a torus.

Briefly, the rest of the proof is as follows: Isotope $G$ into a "good position" and examine the transverse intersections of $G$ with the fibres. From these, extract a sequence of curves which provide a path in the curve complex of the fibre. This path gives the desired bound. Here are the details.

Applying a theorem of Thurston [24], isotope $G$ until all non-transverse intersections with the fibres occur at a finite number of saddle tangencies. Furthermore, there is at most one tangency between $G$ and any fibre $F(\theta)$.

It follows that every transverse curve of intersection between $G$ and $F(\theta)$ is essential in both surfaces. Any transverse curve failing this would, perforce, be inessential in both. But that would lead directly to a center tangency between $G$ and some fibre.

Let $\left\{\theta_{i}\right\}_{i=0}^{n-1}$ be the critical angles where $G$ fails to be transverse to $F\left(\theta_{i}\right)$. Every critical angle gives a saddle for $G$. It follows that $n=-\chi(G) \geq 2$ as $G$ is orientable and not a torus. Pick regular angles $\left\{r_{i}\right\}_{i=0}^{n-1}$ where $\theta_{i-1}<$ $r_{i}<\theta_{i}$, with indices taken $\bmod n$. Apply a rotation to force $r_{0}=0=2 \pi$. Let $\alpha_{i}$ be any curve component of $F\left(r_{i}\right) \cap G$ and recall that $\alpha_{i} \subset F\left(r_{i}\right)$ is essential.

As in Figure 1, the curve $\alpha_{i+1}$ may be isotoped back through the submanifold $F \times\left[r_{i}, r_{i+1}\right]$ to lie on the fibre $F\left(r_{i}\right)$, disjointly from $\alpha_{i}$. (To see that this is correct: let $\Sigma$ be the singular component of $F\left(\theta_{i}\right) \cap G$; the component of $F\left(\theta_{i}\right) \cap G$ which is not a simple closed curve. So $\Sigma$ is homeomorphic to a graph with one vertex and two edges. Let $N=F \times\left[r_{i}, r_{i+1}\right]$ and let $P$ be the component of $G \cap N$ which contains $\Sigma$. As $G$ is orientable and $P$ has at least two boundary components, $P$ is homeomorphic to a "pair-of-pants"; a two-sphere where three open disks (with disjoint closures) have been removed. Note that $P$ is properly embedded in $N$ with essential boundary in $\partial N$. Thus, $P$ is incompressible in $N$. A standard argument shows that $P$ 


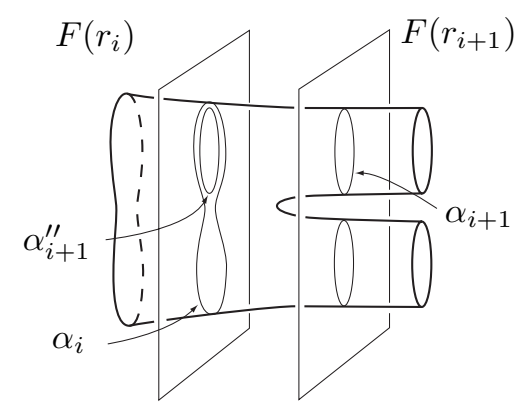

Figure 1: Isotoping $\alpha_{i+1}$ back to the curve $\alpha_{i+1}^{\prime \prime} \subset F\left(r_{i}\right)$.

is boundary-compressible. Boundary-compressing $P$ gives one or two annuli properly embedded in $N$, at least one of which is horizontal; isotopic rel boundary to an annulus of the form $\beta \times\left[r_{i}, r_{i+1}\right]$. So there is either a horizontal annulus giving an isotopy of $\alpha_{i}$ forward into $F\left(r_{i+1}\right)$ or a horizontal annulus giving an isotopy of $\alpha_{i+1}$ back into $F\left(r_{i}\right)$. In either case, deduce the desired result.)

In this fashion, produce a sequence of curves $\left\{\alpha_{i}^{\prime}\right\}_{i=0}^{n}$ (indices not taken $\bmod n)$ in $F\left(r_{0}\right)=F(0)$ such that

- $\alpha_{i}^{\prime}$ is isotopic to $\alpha_{i}$ through $F \times\left[0, r_{i}\right]$ for $i \in\{1,2, \ldots, n-1\}$,

- $\alpha_{0}^{\prime}=\alpha_{0}$,

- $\alpha_{n}^{\prime}$ is obtained by isotoping $\alpha_{0}$ off of $F(2 \pi)$ back through $F \times[0,2 \pi]$,

- $\alpha_{i}^{\prime} \cap \alpha_{i+1}^{\prime}=\emptyset$ for $i=0,1, \ldots, n-1$, and

- $\varphi\left(\alpha_{n}^{\prime}\right)$ is isotopic to $\alpha_{0}^{\prime}$.

Thus,

$$
d_{\mathcal{C}}(\varphi) \leq d_{\mathcal{C}}\left(\alpha_{n}^{\prime}, \varphi\left(\alpha_{n}^{\prime}\right)\right) \leq n=-\chi(G)
$$

and the theorem is proven.

Theorems 6.1 and 3.1 have direct consequences:

Corollary 3.2. Any Heegaard splitting $H \subset M(\varphi)$ satisfying $-\chi(H)<$ $d_{\mathcal{C}}(\varphi)$ is a stabilization of the standard splitting.

Before proving this, a few remarks are in order. 
Remark 3.3. When the translation distance of $\varphi$ is bigger than $4 g(F)$, the standard splitting is the unique minimal genus splitting, up to isotopy.

Corollary 3.4. Suppose that $\varphi$ is pseudo-Anosov. If $n \in \mathbb{N}$ is sufficiently large, then the standard Heegaard splitting of $M\left(\varphi^{n}\right)$ is the unique splitting of minimal genus, up to isotopy.

This follows from our Corollary 3.2 and Lemma 4.6 of [13] which states that $d_{\mathcal{C}}\left(\varphi^{n}\right)$ grows linearly with $n$.

Remark 3.5. Rubinstein [16] has obtained Corollary 3.4 using techniques from minimal surface theory. Lackenby [11], using similar ideas, has obtained a slightly weaker result. Note also that a closed hyperbolic surface bundle $M(\varphi)$ is covered by the bundle $M\left(\varphi^{n}\right)$. Thus, Corollary 3.4 may be considered as weak evidence for a "yes" answer to a question of Boileau; see Problem 3.88 in [9].

Proof of Corollary 3.2. Let $H \subset M(\varphi)$ be a Heegaard splitting with $-\chi(H)<d_{\mathcal{C}}(\varphi)$. If $H$ is stabilized, we may destabilize to obtain a Heegaard splitting with smaller $-\chi$. So, assume that $H$ is not stabilized. As $M(\varphi)$ is irreducible, it follows that $H$ is irreducible [25].

Now, by Theorem 6.1, the splitting $H$ cannot be strongly irreducible. Thus, $H$ is simultaneously weakly reducible and irreducible. Following [22] (to be precise, the final sentence of Remark 2.3 of that paper), there are disjoint disk systems $\mathbf{D} \subset V$ and $\mathbf{E} \subset W$ with the following property: compressing $H$ along $\mathbf{D} \cup \mathbf{E}$ yields a non-empty incompressible surface $G$. This surface need not be connected. Also note that $-\chi(G)<-\chi(H)$.

If a component of $G$ is not isotopic to the fibre $F$, then apply Theorem 3.1 to find either $d_{\mathcal{C}}(\varphi) \leq 1$ or $d_{\mathcal{C}}(\varphi) \leq-\chi(G)$. The first inequality, combined with our assumption $-\chi(H)<d_{\mathcal{C}}(\varphi)$, implies that the surface bundle $M(\varphi)$ is either the three-sphere or a lens-space. This is a contradiction as $\pi_{1}(M(\varphi))$ contains a non-Abelian surface group. The second inequality combined with our assumption that $-\chi(H)<d_{\mathcal{C}}(\varphi)$ implies $-\chi(H)<-\chi(H)$, an impossibility.

It follows that $G$ is isotopic to a collection of fibres. Recall that the surface $G$ was obtained via compressing a separating surface. Thus, $G$ itself must be separating. Hence, $G$ is the union of an even number of fibres. Thus, the genus of $H$ is at least that of the standard splitting. (Note that even this much of the proof establishes, when $d_{\mathcal{C}}(\varphi)>4 g(F)$, that the standard splitting has minimal genus.) 
Let $N \cong F \times I$ be the closure of some component of $M \backslash G$. Let $\mathbf{A}$ be the compressing disks of $\mathbf{D} \cup \mathbf{E}$ contained in $N$. Now, let $H^{\prime} \subset N$ be the surface obtained by compressing $H$ along all disks of $(\mathbf{D} \cup \mathbf{E}) \backslash \mathbf{A}$. Finally, let $H^{\prime \prime}$ be the component of $H^{\prime}$ contained in $N$.

Then $H^{\prime \prime}$ is an irreducible Heegaard splitting for $N$ such that both boundary components of $N$ lie on the same side of $H^{\prime \prime}$ (see Lemma 2.4 of [22]). By Theorem 2.9, it follows that $H^{\prime \prime}$ is the standard type 2 splitting of $N \cong F \times I$. The identical argument applies to all other components of $M(\varphi) \backslash G$. We conclude that $H$ is obtained by amalgamation of these splittings (Proposition 2.8 of [22]).

Now, if $|G| \geq 4$, a pleasant exercise shows that $H$ was stabilized - a contradiction. It follows that $H$ is the standard splitting of $M(\varphi)$ and this completes the proof.

\section{Analyzing intersections.}

Briefly, the proof of Theorem 6.1 is as follows: Isotope the splitting surface $H$ into a "good position" and examine the transverse intersections of $H$ with the fibres. From these, extract a sequence of curves which provide a path in the curve complex of the fibre. This path gives the desired bound. As might be expected, the details are more delicate than in the proof of Theorem 3.1. In particular, a replacement for Thurston's theorem is needed.

The rest of this section explores the nature of intersections between a Heegaard splitting surface and the fibres of a surface bundle over the circle.

Fix a surface automorphism $\varphi: F \rightarrow F$. Recall that $\pi_{F}: M(\varphi) \rightarrow S^{1}$ is the associated map realizing $M(\varphi)$ as a bundle over the circle. Let $H \subset$ $M(\varphi)$ be a Heegaard splitting. Pick a fibre $F(\theta)=\pi_{F}^{-1}(\theta)$ which meets $H$ transversely.

Definition 4.1. A simple closed curve component $\alpha \subset F(\theta) \cap H$ is noncompressing if $\alpha$ is either essential in both surfaces or inessential in both. We call the curve $\alpha$ mutually essential if $\alpha$ is essential in both surfaces. Finally, $\alpha$ is mutually inessential if $\alpha$ is inessential in both surfaces.

Note that, as $F(\theta)$ is incompressible, no curve of intersection may be essential in $F(\theta)$ while being inessential in the splitting surface.

Wiggle $H$ slightly so that $\pi_{F} \mid H$ is Morse; i.e. has singularities of Morse type. Let $p \in H$ be a saddle critical point of $\pi_{F} \mid H$. Let $\theta=\pi_{F}(p)$ and let $P$ be the component of $H \cap \pi_{F}^{-1}[\theta-\epsilon, \theta+\epsilon]$ containing $p$. 
Definition 4.2. If every component of $\partial P$ is mutually essential, we call $p$ an essential saddle. If every component of $\partial P$ is non-compressing, but at least one is inessential in $H$, then we call $p$ an inessential saddle.

Lemma 4.3. Fix a Heegaard splitting $H \subset M(\varphi)$ so that $\pi_{F} \mid H$ is Morse. If $F^{\prime}$ is isotopic to the fibre $F(\theta)$, the surface $H^{\prime}$ is isotopic to $H$, and $F^{\prime}$ is transverse to $H^{\prime}$, then $F^{\prime} \cap H^{\prime}$ contains at least one curve which is not a mutually inessential curve.

Proof. Suppose that $F^{\prime} \cap H^{\prime}$ meet only in mutually inessential curves. Then, by an innermost disk argument, there is a further isotopy making them disjoint. This cannot be, as handlebodies do not contain closed embedded incompressible surfaces.

Lemma 4.4. Fix $H \subset M(\varphi)$ so that $\pi_{F} \mid H$ is Morse. Suppose angles $\theta_{-}<$ $\theta_{+}$are given such that:

- The splitting $H$ intersects $F\left(\theta_{ \pm}\right)$transversely.

- For every angle $\theta \in\left[\theta_{-}, \theta_{+}\right]$all simple closed curve components of $F(\theta) \cap H$ are non-compressing.

- Every saddle of $\pi_{F} \mid H$ in $F \times\left[\theta_{-}, \theta_{+}\right]$is inessential.

Then there is a curve of $F\left(\theta_{-}\right) \cap H$, essential in $F\left(\theta_{-}\right)$, which is isotopic, through $F \times\left[\theta_{-}, \theta_{+}\right]$, to a curve of $F\left(\theta_{+}\right) \cap H$.

Proof. Let $\left\{\theta_{i}\right\}$ be the critical angles of $\pi_{F} \mid H$ which lie in $\left[\theta_{-}, \theta_{+}\right]$. Choose $r_{i}$ slightly greater than the $\theta_{i}$ and let $R=\left\{r_{i}\right\} \cup\left\{\theta_{-}+\epsilon\right\}$ be a set of regular angles.

For every $r \in R$ surger $H$ along every curve of $F(r) \cap H$ which bounds a disk in $F(r)$, innermost first. Let $H^{\prime}$ be the intersection of the surgered surface with $F \times\left[\theta_{-}, \theta_{+}\right]$. Note that $\pi_{F} \mid H^{\prime}$ has exactly two new critical points for every surgery curve. See Figure 2.

Claim. The surface $H^{\prime}$ is a union of spheres, disks, and annuli. Every annulus component has boundary which is essential in one of the $F\left(\theta_{ \pm}\right)$.

Proof. Fix attention on a component $H^{\prime \prime} \subset H^{\prime}$. Begin by examining the critical points of $\pi_{F} \mid H^{\prime \prime}$ and drawing conclusions about the Euler characteristic of $H^{\prime \prime}$. 


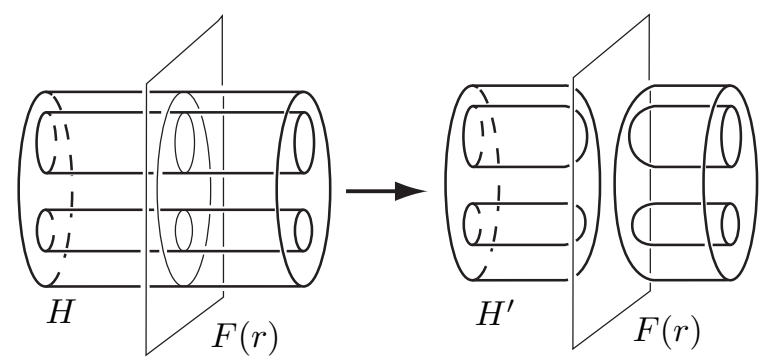

Figure 2: Constructing $H^{\prime}$ from $H$.

If $\pi_{F} \mid H^{\prime \prime}$ has no critical points then $H^{\prime \prime}$ is a horizontal annulus. In this case the boundary of $H^{\prime \prime}$ must be essential in its fibre, by the construction of $H^{\prime}$. Note that this kind of situation is the desired conclusion of the lemma at hand.

If $\pi_{F} \mid H^{\prime \prime}$ has more critical points of even index than odd then $H^{\prime \prime}$ is a disk or sphere.

Now, suppose $p \in H^{\prime \prime}$ is a critical point of saddle type and let $\pi_{F}(p)=\theta$. Let $P$ be the component of $H \cap \pi_{F}^{-1}[\theta-\epsilon, \theta+\epsilon]$ meeting $p$.

As $p$ is not an essential saddle at least one boundary component of $P$ is inessential in its fibre. If all three are inessential then $H^{\prime \prime}$ is a two-sphere. If exactly two components of $\partial P$ are inessential in their fibres then one of the two surfaces $F(\theta \pm \epsilon)$ is compressible in $F \times\left[\theta_{-}, \theta_{+}\right]$, a contradiction. Assume, therefore, that exactly one component of $\partial P$ is inessential in the containing fibre. Call this curve $\alpha$.

Assume that $\pi_{F}(\alpha)=\theta-\epsilon$; that is, the inessential curve $\alpha$ lies to the left of the saddle point. (The other case is handled similarly.) Let $r \in R$ be the regular value appearing just before the critical value $\theta=\pi_{F}(p)$. That is, $r<\theta-\epsilon<\theta$. Note that $\pi_{F} \mid H$ has no critical values between $r$ and $\theta$. See Figure 3.

Deduce that, in the surface $H$, there is a horizontal annulus isotopic to $\alpha \times[r, \theta-\epsilon]$. Thus the surface $H^{\prime \prime}$ has a center tangency with the fibre just before the angle $r$. This gives a disk capping off the curve $\alpha$. Again, see Figure 3. It follows that every saddle in $H^{\prime \prime}$ is paired with at least one critical point of even index. Thus, if not a sphere or disk, $H^{\prime \prime}$ is an annulus.

Finally, if $H^{\prime \prime}$ is an annulus then $H^{\prime \prime}$ has two boundary components. By construction, each of these is an essential curve in one of the surfaces $F\left(\theta_{ \pm}\right)$. This finishes the claim. 


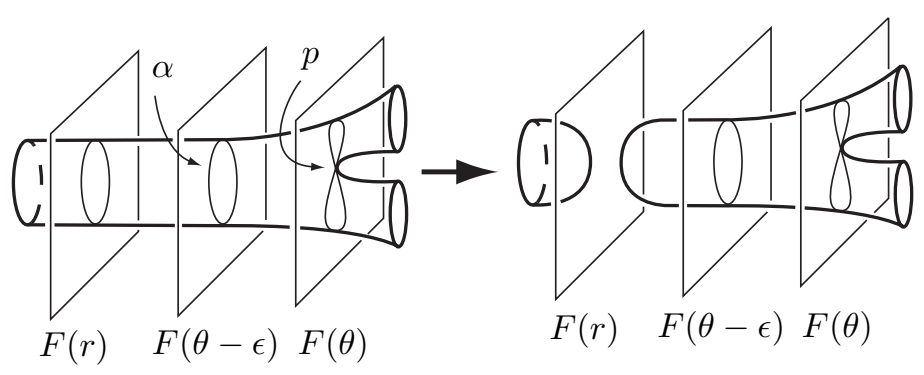

Figure 3: A surgery just before $\alpha$

Now to complete the proof of the lemma. Suppose that no component of $H^{\prime}$ meets both boundary components of $F \times\left[\theta_{-}, \theta_{+}\right]$. Thus, by the claim, every component of $H^{\prime}$ meeting $F\left(\theta_{-}\right)$is boundary parallel in $F \times\left[\theta_{-}, \theta_{+}\right]$. Isotope $F\left(\theta_{-}\right)$across these boundary-parallelisms to obtain a surface $F^{\prime}$ which intersects the splitting surface $H$ only in mutually inessential curves. This contradicts Lemma 4.3.

So there is a component $H^{\prime \prime} \subset H^{\prime}$ which meets both $F\left(\theta_{-}\right)$and $F\left(\theta_{+}\right)$. By the claim above this $H^{\prime \prime}$ must be isotopic to a horizontal annulus with boundary essential in the containing fibres. The lemma is proved.

\section{The graphic and its labellings.}

To begin, this section discusses height functions subordinate to a Heegaard splitting. We then compare one of these to a bundle structure to obtain a "graphic" in the sense of Rubinstein and Scharlemann [17]. This technique is also similar that of [6]. We also refer the reader to [10] as an informative paper on this topic.

Fix attention now on a Heegaard splitting $H$ of a closed, orientable, three-manifold $M$. Recall that $H$ cuts $M$ into a pair of handlebodies $V$ and $W$.

\subsection{Height functions.}

Choose a diffeomorphism between the handlebody $V$ and a regular neighborhood of a connected, finite, polygonal graph $\Theta \subset \mathbb{R}^{3}$. For simplicity, assume that every vertex of $\Theta$ has valence two or three. Let $\Theta_{V}$ be the image of $\Theta$ inside of $V$. Any such $\Theta_{V}$ is a spine of $V$. 
Definition 5.1. A smooth map $\pi_{H}: M \rightarrow I$ is a height function with respect to the splitting $H$ if

- the level $H(t)=\pi_{H}^{-1}(t)$ is isotopic to $H$ for all $t \in(0,1)$,

- the graphs $\Theta_{V}=\pi_{H}^{-1}(0), \Theta_{W}=\pi_{H}^{-1}(1)$ are spines for $V$ and $W$,

- there is a map $h: H \times I \rightarrow M$ (a sweep-out) such that

- $h \mid H \times(0,1)$ is a diffeomorphism,

$-\pi_{H} \circ h$ is projection onto the second factor,

- for small $\epsilon$, the image of $h \mid H \times[0, \epsilon]$ gives the structure of a mapping cylinder to $V(\epsilon)=\pi_{H}^{-1}[0, \epsilon]$, for some deformation retraction $\partial V(\epsilon) \rightarrow \Theta_{V}$

- the previous condition also holds for the handlebody $W(1-\epsilon)=$ $\pi_{h}^{-1}[1-\epsilon, 1]$.

The last two conditions on the sweep-out ensure that if an embedded surface $F \subset(M, H)$ meets $\Theta_{V}$ (or $\Theta_{W}$ ) transversely, then $F \cap V(\epsilon)$ (or $F \cap W(1-\epsilon))$ is a collection of properly embedded disks.

\subsection{The graphic.}

Let $H \subset M(\varphi)$ be a Heegaard splitting of the given surface bundle. Choose a bundle map, $\pi_{F}$, and a height function, $\pi_{H}$, as above and insist that the two functions are generic with respect to each other. Define the map $\pi_{\Gamma}: M(\varphi) \rightarrow S^{1} \times I$ by $\pi_{\Gamma}(x)=\left(\pi_{F}(x), \pi_{H}(x)\right)$. As a bit of terminology, we sometimes call $\pi_{\Gamma}(F(\theta))$ a vertical interval and call $\pi_{\Gamma}(H(t))$ a horizontal circle. Also, let $\Gamma(\theta, t)=\pi_{\Gamma}^{-1}(\theta, t)=F(\theta) \cap H(t)$.

The graphic of the map $\pi_{\Gamma}=\left(\pi_{F}, \pi_{H}\right)$ is the set

$$
\Lambda=\overline{\left\{(\theta, t) \in S^{1} \times(0,1) \mid F(\theta) \text { is not transverse to } H(t)\right\}}
$$

where the closure is taken in $S^{1} \times I$. As in the papers cited above: $\Lambda$ is a graph with smooth edges meeting the boundary of the annulus transversely.

Definition 5.2. The open cells of $S^{1} \times(0,1) \backslash \Lambda$ are called the regions of the graphic.

If $(\theta, t)$ is a point of a region, then $\Gamma(\theta, t)=F(\theta) \cap H(t)$ is a collection of simple closed curves embedded in $M(\varphi)$. 
Remark 5.3. Suppose that $(\theta, t),\left(\theta^{\prime}, t^{\prime}\right)$ are two points in the same region. Then the combinatorics of $\Gamma(\theta, t)$ and $\Gamma\left(\theta^{\prime}, t^{\prime}\right)$ are identical, as follows:

Suppose that $\alpha$ is a short horizontal or vertical arc embedded in the interior of a region $R$. Suppose also that $(\theta, t),\left(\theta^{\prime}, t^{\prime}\right)$ are the endpoints of $\alpha$. Let $\gamma$ be a component of $\Gamma(\theta, t)$. Then there is an ambient isotopy taking $\gamma$ to $\gamma^{\prime} \subset \Gamma\left(\theta^{\prime}, t^{\prime}\right)$ supported in a neighborhood of an annulus of $F(\theta)$ (if $\alpha$ is vertical) or an annulus of $H(t)$ (if $\alpha$ is horizontal). Furthermore, this ambient isotopy may be chosen to take $F(\theta)$ to $F\left(\theta^{\prime}\right)$ and $H(t)$ to $H\left(t^{\prime}\right)$.

By crawling along horizontal and vertical arcs, any pair of points in $R$ may be joined. Thus, most properties of $\Gamma(\theta, t)$ depend only on the region containing $(\theta, t)$.

As above, the edges are the one-dimensional strata of $\Lambda$.

Remark 5.4. When $(\theta, t)$ lies on an edge, there is one component, $\Sigma(\theta, t)$, of $\Gamma(\theta, t)$ which is not a simple closed curve. This $\Sigma$ is the singular component. The name of the point $(\theta, t)$ is omitted when clear from context.

There are two kinds of edges: those representing a center tangency between a fibre and a level surface, and those representing a saddle tangency. Crossing an edge of the graphic from region $R$ to $R^{\prime}$ causes the combinatorics of the curves to change. If the edge represents a center tangency, then a single curve of $R$ disappears (appears). It follows that this curve is mutually inessential; in other words, it bounds a disk in both the fibre and the level. In this situation, $\Sigma$ is a single point.

If the edge represents a saddle, then two curves of $R$ touch as the edge is crossed and become a single curve of $R^{\prime}$ (or the reverse). When $(\theta, t)$ lies on such an edge, the singular component $\Sigma$ is a four-valent graph with one vertex, embedded in both the fibre $F(\theta)$ and the level $H(t)$.

The vertices are the zero-dimensional strata of the graphic $\Lambda$.

Remark 5.5. There are several possibilities for a vertex $v=(\theta, t)$ :

1. All vertices of valence one occur at height 0 or 1 .

2. A vertex with valence two in $\Lambda$ is a birth-death vertex. Both edges lie in the same quadrant with respect to the vertex. (As in [10].) See Figure 4.

3. A vertex of valence four is a crossing vertex. Here, the four edges lie in distinct quadrants and the tangent directions of opposite edges agree. The edges cut a small neighborhood of the vertex into four regions. Again, see Figure 4. There are two further subcases: 
- If there are two singular components $\Sigma, \Sigma^{\prime} \subset \Gamma(\theta, t)$, then $v$ is a disjoint crossing vertex.

- If there is a single singular component $\Sigma$, then $v$ has entangled saddles. In this case, $\Sigma$ is a four-valent graph with exactly two vertices.
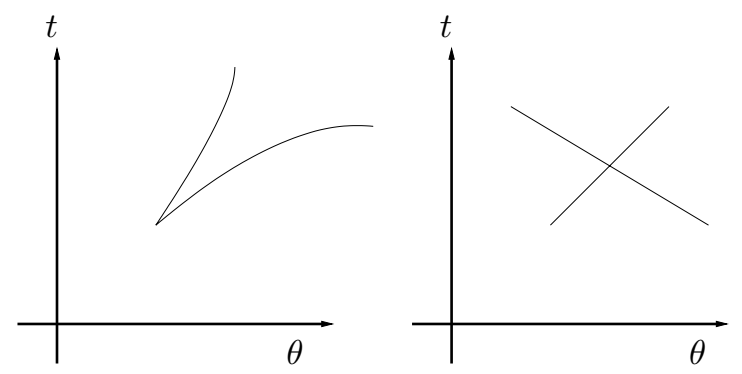

Figure 4: A birth-death vertex and a crossing vertex.

Finally, general position implies that for every angle $\theta$, the vertical interval $\pi_{\Gamma}(F(\theta))=\{\theta\} \times I$ meets the graphic $\Lambda$ at most once non-transversely, either at a vertex or at a tangency with the interior of an edge. The same holds for horizontal circles $\pi_{\Gamma}(H(t))=S^{1} \times\{t\}$.

\subsection{Labellings.}

The following labellings will play an important role in the proof of Theorem 6.1. Recall that the level surface $H(t)$ cuts $M(\varphi)$ into a pair of handlebodies $V(t)$ and $W(t)$.

Definition 5.6. For each $t \in(0,1)$, the level $H(t)$ is labelled $\mathbb{V}$ (or $\mathbb{W}$ ) if there is a value $\theta \in[0,2 \pi]$ and non-singular simple closed curve component $\gamma \subset \Gamma(\theta, t)=F(\theta) \cap H(t)$ which bounds an essential disk in $V(t)$ (or $W(t)$ ).

A slightly finer labelling is also required.

Definition 5.7. A region $R$ is labelled with a $\mathbb{V}$ (or $\mathbb{W}$ ) if there is a $(\theta, t) \in R$ and a component $\gamma \subset \Gamma(\theta, t)$ such that $\gamma$ bounds an essential disk in $V(t)$ $(W(t))$.

As we shall see in the proof below, each level and region receives at most one label (or none at all) and these labels reveal information about the relative positions of the fibre and level surface. 


\section{Proof of the main theorem.}

A sequence of claims will now prove:

Theorem 6.1. If $H \subset M(\varphi)$ is a strongly irreducible Heegaard splitting, then the translation distance of $\varphi$ is at most the negative Euler characteristic of $H$. That is,

$$
d_{\mathcal{C}}(\varphi) \leq-\chi(H)
$$

Pick a bundle map $\pi_{F}: M(\varphi) \rightarrow S^{1}$ and a height function $\pi_{H}: M(\varphi) \rightarrow$ $I$ which respect the given fibre and splitting. Recall that $\Theta_{V}=\pi_{H}^{-1}(0)$ and $\Theta_{W}=\pi_{H}^{-1}(1)$ are spines for the handlebodies $V$ and $W$. Also, the levels $H(t)=\pi_{H}^{-1}(t)$ impose a product structure on $M(\varphi) \backslash\left(\Theta_{V} \cup \Theta_{W}\right)$.

As above, obtain a graphic $\Lambda$ in the annulus $S^{1} \times I$. We begin by obtaining a few fairly standard facts about the labellings defined in the previous section.

\subsection{Analyzing the labelling.}

Claim 6.2. For all sufficiently small positive values $\epsilon$, the level $H(\epsilon)$ is labelled $\mathbb{V}$ while $H(1-\epsilon)$ is labelled $\mathbb{W}$.

This follows directly from the construction of the height function and genericity.

Claim 6.3. No level or region is labelled with both $a \mathbb{V}$ and $a \mathbb{W}$. Also, if a region $R$ is labelled, then every level $H(t)$, such that $\pi_{\Gamma}(H(t)) \cap R \neq \emptyset$, receives the same label. Finally, if a level $H(t)$ is labelled, then some region meeting $\pi_{\Gamma}(H(t))$ receives the same label.

This follows from strong irreducibility, the fact that the curves $\Gamma(\theta, t)=$ $F(\theta) \cap H(t)$, as $\theta$ varies, form a singular foliation of $H(t)$, and Remark 5.3.

Remark 6.4. Suppose that $H(t)$ is labelled and $\gamma \subset \Gamma(\theta, t)$ is a witness of this fact. Then there is a $\gamma^{\prime} \subset \Gamma\left(\theta^{\prime}, t\right)$ which is also a witness, for all $\theta^{\prime}$ sufficiently close to $\theta$.

Remark 6.5. Suppose $H(t)$ is labelled with a $\mathbb{V}$ (or $\mathbb{W}$ ). By incompressibility of $F(\theta)$, the given curve $\gamma$ bounds a disk $D \subset F(\theta)$. By the "no nesting" lemma, Lemma 2.8, $D$ is isotopic rel $\gamma$ to a disk properly embedded in $V(t)$ (or $W(t)$ ). 
From Remark 5.4, deduce:

Claim 6.6. If two regions are both adjacent to an edge representing a center tangency, then both regions have the same label.

The next claim is not strictly required for the proof of Theorem 6.1. We include it both to simplify our proof of the theorem and to shed light on the general situation.

Claim 6.7. Suppose that $H\left(t^{\prime}\right)$ is labelled $\mathbb{V}$. Suppose $0<t<t^{\prime}$. Then $H(t)$ is labelled $\mathbb{V}$ as well. Identically, if $t^{\prime}<t<1$ and $H\left(t^{\prime}\right)$ is labelled $\mathbb{W}$, then $H(t)$ is labelled $\mathbb{W}$.

Proof. Consider the case where $H\left(t^{\prime}\right)$ is labelled $\mathbb{V}$. By hypothesis, we are given an angle $\theta^{\prime}$ and a curve $\gamma^{\prime \prime} \subset \Gamma\left(\theta^{\prime}, t^{\prime}\right)$ bounding an essential disk in $V\left(t^{\prime}\right)$. We may choose an angle $\theta$ close to $\theta^{\prime}$ so that firstly, by Remark 6.4 , there is a curve $\gamma^{\prime} \subset \Gamma\left(\theta, t^{\prime}\right)$ also bounding an essential disk in $V\left(t^{\prime}\right)$ and secondly, by general position, the point $(\theta, t)$ lies in a region of the graphic. That is, $(\theta, t) \notin \Lambda$.

By Remark 6.5, the curve $\gamma^{\prime}$ bounds a disk $D^{\prime} \subset F(\theta)$. Furthermore, choosing a different $\gamma^{\prime} \subset \Gamma\left(\theta, t^{\prime}\right)$ if necessary, assume that $\gamma^{\prime}$ is "innermost" in the sense that all curves of interior $\left(D^{\prime}\right) \cap H\left(t^{\prime}\right)$ are inessential in $H\left(t^{\prime}\right)$.

Let $\Gamma\left(D^{\prime}, t\right)=D^{\prime} \cap H(t) \subset \Gamma(\theta, t)$. Suppose that all components of $\Gamma\left(D^{\prime}, t\right)$ are inessential in $H(t)$. Isotope $D^{\prime}$ rel $\gamma^{\prime}$ off of $H(t)$ to a disk $E$ with $E \cap V(t)=\emptyset$. Let $N=\overline{V\left(t^{\prime}\right) \backslash V(t)}$ and note that $N \cong H \times I$ while $\partial N=H(t) \cup H\left(t^{\prime}\right)$. Thus, we may further isotope $E$ rel $\gamma^{\prime}$ out of $\operatorname{interior}(N)$. This pushes $E$ to a disk $E^{\prime} \subset W\left(t^{\prime}\right)$. Thus, $\gamma^{\prime}$ either bounds an essential disk in $W\left(t^{\prime}\right)$ or is trivial in $H\left(t^{\prime}\right)$. The first implies that $H$ is reducible while the second contradicts our choice of $\gamma^{\prime}$.

Thus, there is a curve, $\gamma \subset \Gamma\left(D^{\prime}, t\right)$, which is essential in $H(t)$ and is the innermost such in $D^{\prime}$. Let $D \subset D^{\prime}$ be the disk which $\gamma$ bounds. Recall that all curves of interior $(D) \cap \partial N$ are inessential in $\partial N=\partial\left(\overline{V\left(t^{\prime}\right) \backslash V(t)}\right)$. So, there is an isotopy of $D$ rel $\gamma$ to a disk lying inside of $M(\varphi) \backslash\left(H\left(t^{\prime}\right) \cup H(t)\right)$.

Now, if this disk lies in $N$, then $\gamma$ could not be essential in $H(t)$. It follows that $\gamma$ bounds a disk in $V(t)$. Thus, $H(t)$ is labelled with a $\mathbb{V}$, as claimed.

As a bit of notation, set $L(V)=\{t \in(0,1) \mid H(t)$ is labelled $\mathbb{V}\}$ and $L(W)=\{t \in(0,1) \mid H(t)$ is labelled $\mathbb{W}\}$. Define $t_{V}=\sup L(V)$ and $t_{W}=$ $\inf L(W)$ 
Claim 6.8. The sets $L(V)$ and $L(W) \subset(0,1)$ are non-empty, disjoint, connected, and open. Also, $t_{V} \leq t_{W}$.

Proof. The first sentence follows from Claims 6.2, 6.3, 6.7, and the fact that if $\gamma \subset \Gamma(\theta, t)$ is a non-singular component. then there are $\gamma^{\prime} \subset \Gamma(\theta, t-\epsilon)$ and $\gamma^{\prime \prime} \subset \Gamma(\theta, t+\epsilon)$ with combinatorics identical to $\gamma$. The second follows from the fact that $L(V), L(W)$ are disjoint open intervals and that inf $L(V)=0$ by Claim 6.2.

It follows that if $t_{V} \leq t_{0} \leq t_{W}$, then $H\left(t_{0}\right)$ is unlabelled.

\subsection{Analyzing the unlabelled level.}

The unlabelled level $H\left(t_{0}\right)$ found above will serve as a replacement for Thurston's theorem used in the proof of Theorem 3.1. There are two cases to consider: $t_{V}<t_{W}$ or $t_{V}=t_{W}$.

\subsection{Unlabelled interval.}

Suppose that $t_{V}<t_{W}$. Pick a level $H\left(t_{0}\right)$ which avoids the vertices of the graphic, which is not tangent to any edge of the graphic, and has $t_{V}<$ $t_{0}<t_{W}$. It immediately follows that for every $\theta$ and for every non-singular $\gamma \subset \Gamma\left(\theta, t_{0}\right)$, the curve $\gamma$ is non-compressing - either essential in both $F(\theta)$ and $H\left(t_{0}\right)$ or inessential in both. In the terminology of RubinsteinScharlemann [17], the level $H\left(t_{0}\right)$ is compression-free with respect to every fibre.

We now come to the heart of this case: Let $\left\{\theta_{i}\right\}_{i=0}^{n-1}$ be the critical angles of $\pi_{F} \mid H\left(t_{0}\right)$ corresponding to essential saddles. (As defined in Section 4 , the saddle point $p$ is essential when all boundary components of the associated pair of pants are mutually essential curves.) Choose the indexing so that $\theta_{i}<\theta_{i+1}$ are adjacent.

Suppose first that $n=0$. Rotate the $S^{1}$ coordinate, if necessary, so that $F(0)$ meets $H\left(t_{0}\right)$ transversely. Cut $M(\varphi)$ along $F(0)$. Note that $H\left(t_{0}\right)$, inside of $F \times[0,2 \pi]$, satisfies the hypotheses of Lemma 4.4. Thus, there is a mutually essential curve of $F(0) \cap H\left(t_{0}\right)$ isotopic, through $F \times[0,2 \pi]$, to a curve of $F(2 \pi)$. Thus, $d_{\mathcal{C}}(\varphi) \leq 1<2 \leq-\chi(H)$, as desired.

Suppose now that $n>0$. Choose $\epsilon$ sufficiently small and positive. So, for each critical angle, there is an essential saddle in $H\left(t_{0}\right) \cap\left(F \times\left[\theta_{i}-\epsilon, \theta_{i}+\right.\right.$ $\epsilon])$. The pair of pants given by this saddle contributes -1 to the Euler 
characteristic of $H\left(t_{0}\right)$. Thus, $n \leq-\chi(H)$.

By Lemma 4.4, there are also mutually essential curves $\alpha_{i} \subset \Gamma\left(\theta_{i}+\epsilon, t_{0}\right)$ and $\alpha_{i}^{\prime} \subset \Gamma\left(\theta_{i+1}-\epsilon, t_{0}\right)$ such that $\alpha_{i}^{\prime}$ is isotopic to $\alpha_{i}$ through $F \times\left[\theta_{i}+\right.$ $\left.\epsilon, \theta_{i+1}-\epsilon\right]$. Also, $\alpha_{i}$ may be isotoped back through $F \times\left[\theta_{i}-\epsilon, \theta_{i}+\epsilon\right]$ to lie in $F\left(\theta_{i}-\epsilon\right)$, disjoint from $\alpha_{i-1}^{\prime}$. (This is shown by Figure 1, although the labels will be different.)

As in the proof of Theorem 3.1, the $\alpha_{i}$ give a path of length $n$ in $\mathcal{C}^{1}(F(0))$ starting at $\alpha_{0}$ and ending at $\varphi\left(\alpha_{0}\right)$. This implies $d_{\mathcal{C}}(\varphi) \leq-\chi(H)$, as desired.

\subsection{Only a vertex.}

The more difficult situation occurs when $t_{0}=t_{V}=t_{W}$. By our general position assumption, the horizontal circle $\pi_{\Gamma}\left(H\left(t_{0}\right)\right)$ meets the graphic $\Lambda$ at most once non-transversely.

Claim 6.9. There are regions $R$ and $R^{\prime}$ labelled $\mathbb{V}$ and $\mathbb{W}$, respectively, such that the closures $\bar{R}$ and $\overline{R^{\prime}}$ both meet $\pi_{\Gamma}\left(H\left(t_{0}\right)\right)$. Also, neither $R$ nor $R^{\prime}$ meet $\pi_{\Gamma}\left(H\left(t_{0}\right)\right)$. The horizontal circle $\pi_{\Gamma}\left(H\left(t_{0}\right)\right)$ meets a crossing vertex $\left(\theta_{0}, t_{0}\right)$ of the graphic.

Proof. As $t_{0}=t_{V}$ and by Claim 6.3, there is a region $R$ such that $\bar{R} \cap$ $\pi_{\Gamma}\left(H\left(t_{0}\right)\right)$ is non-empty and $R$ lies below $\pi_{\Gamma}\left(H\left(t_{0}\right)\right)$ in the annulus $S^{1} \times I$. Similarly, there is a region $R^{\prime}$ above the horizontal circle $\pi_{\Gamma}\left(H\left(t_{0}\right)\right)$.

Now, if $\pi_{\Gamma}\left(H\left(t_{0}\right)\right)$ is transverse to the edges of $\Lambda$, then every region $R^{\prime \prime}$ with closure meeting $\pi_{\Gamma}\left(H\left(t_{0}\right)\right)$ also has interior meeting $\pi_{\Gamma}\left(H\left(t_{0}\right)\right)$. Then, as $H\left(t_{0}\right)$ is unlabelled, so are $R$ and $R^{\prime}$. This is a contradiction.

Suppose that $\pi_{\Gamma}\left(H\left(t_{0}\right)\right)$ is only tangent to an edge of the graphic. Then every region, but one, whose closure meets the circle $\pi_{\Gamma}\left(H\left(t_{0}\right)\right)$ also meets $\pi_{\Gamma}\left(H\left(t_{0}\right)\right)$ along its interior. As above, this gives a contradiction. Thus, $\pi_{\Gamma}\left(H\left(t_{0}\right)\right)$ meets a vertex at the point $\left(\theta_{0}, t_{0}\right)$.

Finally, to rule out the possibility that the vertex is a birth-death vertex: When $\pi_{\Gamma}\left(F\left(\theta_{0}\right)\right)$ and $\pi_{\Gamma}\left(H\left(t_{0}\right)\right)$ meet in a birth-death vertex, there are only two edges of the graphic incident on the vertex. As in Figure 4, the slopes of the two edges have the same sign and we may assume that, as the edges leave the vertex, both edges head "northeast." (The other three cases are similar.) Again, every region, but one, whose closure meets the circle $\pi_{\Gamma}\left(H\left(t_{0}\right)\right)$ also meets $\pi_{\Gamma}\left(H\left(t_{0}\right)\right)$ along its interior. Again, this is a contradiction. 
Now, focus attention on this vertex at $\left(\theta_{0}, t_{0}\right)$. Let $\Sigma \subset \Gamma\left(\theta_{0}, t_{0}\right)$ be the union of the singular components. Let $P$ be the components of $H\left(t_{0}\right) \cap$ $\left(F \times\left[\theta_{0}+\epsilon, \theta_{0}-\epsilon\right]\right)$ meeting $\Sigma$. We will call $P$ a foliated regular neighborhood of $\Sigma$, taken in $H\left(t_{0}\right)$. Similarly, let $Q$ be a foliated regular neighborhood of $\Sigma$, taken in $F\left(\theta_{0}\right)$. Finally, let $\partial_{ \pm} P=P \cap F\left(\theta_{0} \pm \epsilon\right)$ while $\partial_{ \pm} Q=Q \cap H\left(t_{0} \pm \epsilon\right)$.

Claim 6.10. The vertex at $\left(\theta_{0}, t_{0}\right)$ has entangled saddles. Also, some component $\beta \subset \partial_{-} Q$ bounds an essential disk in $V\left(t_{0}-\epsilon\right)$ (and some component $\delta \subset \partial_{+} Q$ bounds in $\left.W\left(t_{0}+\epsilon\right)\right)$.

Proof. As $\pi_{\Gamma}\left(F\left(\theta_{0}\right)\right)$ and $\pi_{\Gamma}\left(H\left(t_{0}\right)\right)$ meet in a crossing vertex, there are four regions adjacent with closure meeting the vertex. Call these "north", "east", "south", and "west." See the right-hand side of Figure 4. Again, our general position assumption ensures that $\pi_{\Gamma}\left(H\left(t_{0}\right)\right)$ meets the graphic $\Lambda$ at most once non-transversely. Thus, $\pi_{\Gamma}\left(H\left(t_{0}\right)\right)$ meets $\Lambda$ exactly once nontransversely. Thus, all regions whose closure meets $\pi_{\Gamma}\left(H\left(t_{0}\right)\right)$, other than the north and south, also meet $\pi_{\Gamma}\left(H\left(t_{0}\right)\right)$ along their interior. It follows from Claim 6.3 that all these regions except the south (the region $R$ ) and north $\left(R^{\prime}\right)$ are unlabelled.

Choose a curve $\beta \subset \Gamma\left(\theta_{0}, t_{0}-\epsilon\right)$ which bounds an essential disk in $V\left(t_{0}-\epsilon\right)$. Moving along a straight arc from $\left(\theta_{0}, t_{0}-\epsilon\right)$ to $\left(\theta_{0}+\epsilon, t_{0}\right)$ cannot induce ambient isotopy on $\beta$. If it did, the east region would be labelled with a $\mathbb{V}$, an impossibility.

Thus, the south-east edge represents a saddle tangency. Also, this saddle meets a regular neighborhood of $\beta$, taken inside of $F\left(\theta_{0}\right)$. Symmetrically, the same holds for the south-west edge. We conclude that both saddles lie inside the same component of $\Gamma\left(\theta_{0}, t_{0}\right)$. An identical argument locates $\delta \subset \Gamma\left(\theta_{0}, t_{0}+\epsilon\right)$. It follows that $\Sigma$ is connected, contains two saddle tangencies, and has $\beta$ and $\delta$ as boundary components of $Q$, the vertical foliated neighborhood.

Note that the curves $\beta$ and $\delta$ may be isotoped to curves $\beta_{0}$ and $\delta_{0}$ lying inside of $P$, the regular neighborhood of $\Sigma \subset H\left(t_{0}\right)$. Since $\beta$ and $\delta$ bound disks in $V\left(t_{0}-\epsilon\right)$ and $W\left(t_{0}+\epsilon\right)$, the curves $\beta_{0}$ and $\delta_{0}$ bound disks in $V\left(t_{0}\right)$ and $W\left(t_{0}\right)$.

Remark 6.11. Recall that the Heegaard splitting $H$ and the fibre $F$ both have genus at least two. The graph $\Sigma$ has only two vertices and four edges. Thus, by an Euler characteristic argument, there is an essential simple closed 
curve in $H\left(t_{0}\right)$ disjoint from the subsurface $P$ (and similarly, for $Q \subset F\left(\theta_{0}\right)$ ). So, if $t_{V}=t_{W}$, then the Heegaard splitting $H$ satisfies Thompson's disjoint curve property, defined in [23].

We now examine the properties of the foliated neighborhoods of $\Sigma$; that is, $P \subset H\left(t_{0}\right)$ and $Q \subset F\left(\theta_{0}\right)$. Recall that $\Sigma$ is a connected four-valent graph with two vertices. Let $\rho$ be an edge connecting the two vertices. Let $X$ be a small neighborhood of $\rho$, taken in $H\left(t_{0}\right)$, and let $Y$ be an even smaller neighborhood of $\Sigma$, also taken in $H\left(t_{0}\right)$. Note that $Y \subset P$ and $Y \cong P$. See Figure 5 for a picture of $X \cap Y$. This is combinatorially a 12-gon and $Y \backslash X$ is a union of rectangles in $H\left(t_{0}\right)$. These rectangles glue the $\operatorname{arcs}$ of $(\partial X) \cap Y$ together in pairs.

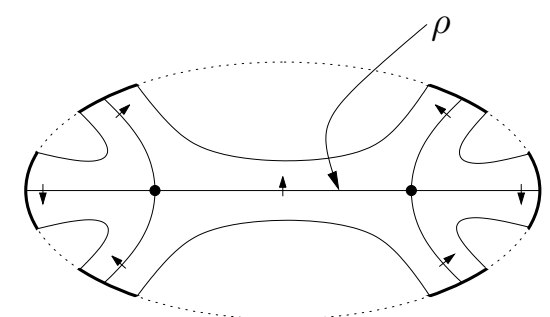

Figure 5: A regular neighborhood of the edge $\rho$ intersecting a regular neighborhood of $\Sigma$.

There are two restrictions on the gluings of these arcs: the orientability of $H$ and the co-orientation of the foliation of $H\left(t_{0}\right)$ by $\Gamma\left(\theta, t_{0}\right)$, as $\theta$ increases. The co-orientation is indicated in Figure 5 by the small arrows. Taking into account symmetry, there are only four possibilities for $Y$, and hence $P$, as shown in the first column of Figure 6 . Note that there are four ways of resolving the two saddles of $\Sigma$; each of these corresponds to moving from the vertex $\left(\theta_{0}, t_{0}\right)$ to one of the four adjacent regions. It follows that resolving the saddles yields the curves $\partial_{ \pm} P$ and $\partial_{ \pm} Q$. Recall that $\beta \subset \partial_{-} Q$ and $\delta \subset \partial_{+} Q$ may be isotoped to $\beta_{0}, \delta_{0} \subset P$, as shown in the second column of Figure 6 .

In the fourth row, there are two possibilities for $\beta_{0}$; either $\beta_{0}$ agrees with $\beta^{\prime}$ or with $\beta^{\prime \prime}$ (the solid curves). The curve $\delta_{0}$ is treated similarly (see the dashed curves). Wherever $\beta_{0}$ and $\delta_{0}$ lie inside the twice-punctured torus, we see that $\beta_{0}$ only meets $\delta_{0}$ once. Thus, the splitting surface $H$ is stabilized, contradicting strong irreducibility. It follows that the foliated neighborhood $P$ is homeomorphic to a four-times punctured sphere, with $\Sigma$ in various 


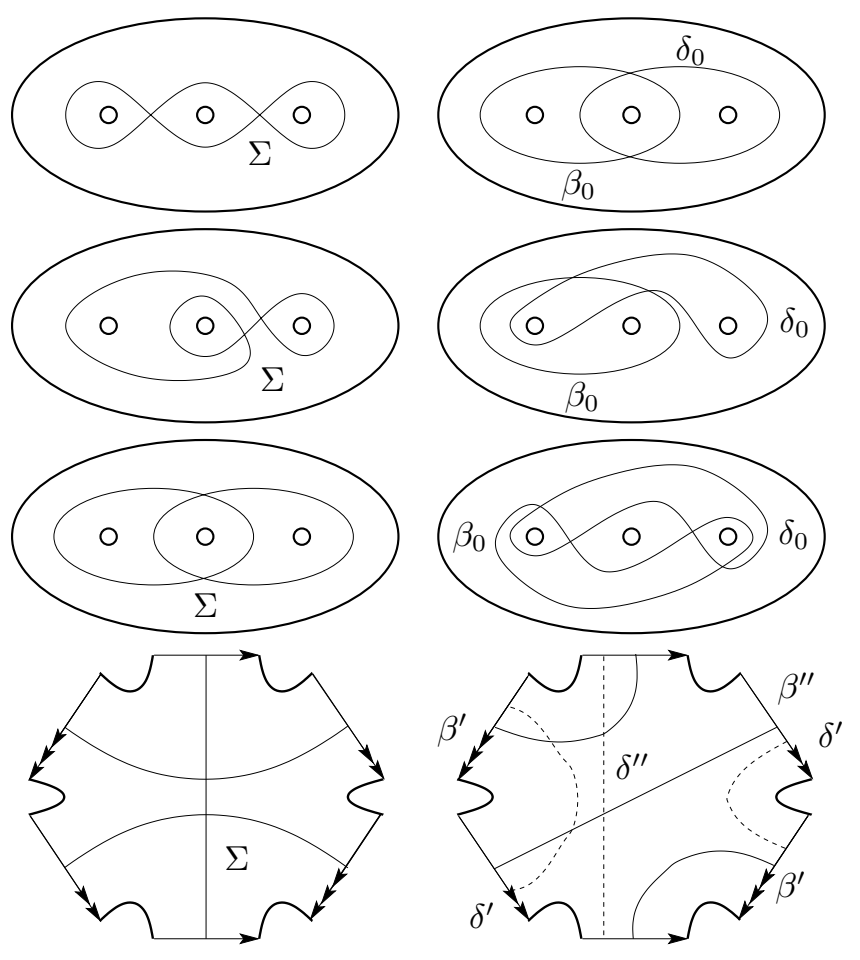

Figure 6: Possibilities for the regular neighborhood $P$.

positions, as shown.

Claim 6.12. Every component of $\partial P$ is essential in $H\left(t_{0}\right)$.

Proof. In each of the three cases, if any component of $\partial P$ bounds a disk in $H\left(t_{0}\right)$, then a component of $\partial P$ bounds a disk in $\overline{H\left(t_{0}\right) \backslash P}$. Isotope $\beta_{0}$ across this disk to make $\beta_{0}$ disjoint from $\delta_{0}$. This contradicts the strong irreducibility of $H$.

Claim 6.13. The components of $\partial P$ are mutually essential curves.

Proof. All are essential in $H\left(t_{0}\right)$ by the above claim. If one of the curves bounds a disk in the containing fibre, then by Lemma 2.8, that curve bounds a disk in $V\left(t_{0}\right)$ or $W\left(t_{0}\right)$. In this case, $H\left(t_{0}\right)$ was labelled with a $\mathbb{V}$ or a $\mathbb{W}$. This contradicts Claim 6.8 and the hypothesis $t_{0}=t_{V}=t_{W}$. 
Now, to carry out an analysis similar to that of the case $t_{V}<t_{W}$. Let $\left\{\theta_{i}\right\}_{i=1}^{n-1}$ be the angles corresponding to the essential saddles of $\pi_{F} \mid H\left(t_{0}\right)$. Let $\left(\theta_{0}, t_{0}\right)$ be the vertex with entangled saddles meeting the horizontal circle $\pi_{\Gamma}\left(H\left(t_{0}\right)\right)$. Choose the indexing so that $\theta_{i}<\theta_{i+1}$ are adjacent. Choose $\epsilon$ sufficiently small and positive. So, for each critical angle, $i>0$, there is an essential saddle in $H\left(t_{0}\right) \cap\left(F \times\left[\theta_{i}-\epsilon, \theta_{i}+\epsilon\right]\right)$ which contributes -1 to the Euler characteristic of $H\left(t_{0}\right)$. As the curves of $\partial P$ are mutually essential (Claim 6.13), the four-punctured sphere $P$ contributes -2 to the Euler characteristic of $H\left(t_{0}\right)$. Altogether, the $n-1$ essential saddles and $P$ contribute $n+1$ to the negative Euler characteristic of $H\left(t_{0}\right)$. That is, $n+1 \leq-\chi(H)$.

By Lemma 4.4, there are also mutually essential curves $\alpha_{i} \subset \Gamma\left(\theta_{i}+\epsilon, t_{0}\right)$ and $\alpha_{i}^{\prime} \subset \Gamma\left(\theta_{i+1}-\epsilon, t_{0}\right)$ such that $\alpha_{i}^{\prime}$ is isotopic to $\alpha_{i}$ through $F \times\left[\theta_{i}+\epsilon, \theta_{i+1}-\right.$ $\epsilon]$. Also, for $i>0, \alpha_{i}$ may be isotoped back through $F \times\left[\theta_{i}-\epsilon, \theta_{i}+\epsilon\right]$ to lie in $F\left(\theta_{i}-\epsilon\right)$, disjoint from $\alpha_{i-1}^{\prime}$. For $i=0$, this last may not hold. Instead, after isotoping $\alpha_{0}$ through $F \times\left[\theta_{0}-\epsilon, \theta_{0}+\epsilon\right]$ to obtain $\alpha_{0}^{\prime \prime}$, both $\alpha_{0}^{\prime \prime}$ and $\alpha_{n-1}^{\prime}$ may lie in a translate of $Q$, the vertical foliated neighborhood of $\Sigma$. As in Remark 6.11, the subsurface $Q$ does not fill $F\left(\theta_{0}-\epsilon\right)$. In any case, $d_{\mathcal{C}}\left(\alpha_{0}^{\prime \prime}, \alpha_{n-1}\right) \leq 2$ when considered in the curve complex of the fibre.

Thus, similar to the proof of Theorem 3.1, the $\alpha_{i}$ give a path of length $n+1$ in the graph $\mathcal{C}^{1}(F(0))$. Therefore, $d_{\mathcal{C}}(\varphi) \leq n+1 \leq-\chi(H)$, as desired. This deals with the case where $t_{V}=t_{W}$ and proves the theorem.

We end here with a few remarks and comments:

Question 6.14. What can be said about the higher genus Heegaard splittings of surface bundles with high translation distance?

Question 6.15. Suppose that $M$ has two distinct surface bundle structures. What can be learned from the graphic induced on $S^{1} \times S^{1}$ ?

Remark 6.16. It seems likely that the techniques of Section 6.1 are sufficiently soft to allow a taut foliation to replace the surface bundle structure. See also Question 9.5 of Calegari's problem list on foliations [4].

Remark 6.17. There is no inequality, as in Theorem 6.1, between the genus of a strongly irreducible splitting of $M(\varphi)$ and the stretch factor of the pseudo-Anosov automorphism $\varphi$. Here is the required construction: Fix $H \subset M(\varphi)$ a genus two, strongly irreducible splitting. (For example, let $M(\varphi)$ be the longitudinal filling on D. Rolfsen's $6_{2}$ knot [15]. As the knot is tunnel number one, let $H$ be the resulting genus two Heegaard splitting.) 
Isotope $H$ until all curves of $F(0) \cap H$ are mutually essential. Let $\gamma \subset$ $\Gamma=F(0) \cap H$ be one component. Let $N(\gamma)$ be a regular neighborhood of $\gamma$. Let $\mu, \lambda \subset \partial N(\gamma)$ be a meridian, longitude pair with $\mu$ bounding a disk in $N(\gamma)$ while $\lambda$ is isotopic to $\partial N(\gamma) \cap H$ (which, in turn, is isotopic to $\partial N(\gamma) \cap F)$. Then $M_{n}$, the $1 / n$ Dehn surgery on $\overline{M \backslash N(\gamma)}$, is still a surface bundle, with monodromy $\varphi_{n}$, say. The stretch factor of $\varphi_{n}$ grows linearly with $n$ (see [12]) while the Heegaard genus of $M_{n}$ remains equal to two. Thus, the minimal genus splitting remains strongly irreducible. This completes the construction.

Remark 6.18. It has been asked whether the main theorem of this paper can be improved to refer to translation distance in the pants complex. (See [2].) It is straight-forward to provide candidate counterexamples in genus two, somewhat similar to the above. A subtle argument, shown to us by Canary and Minsky, then proves that the volumes increase without bound. We hope to provide the details of this construction in a future paper.

\section{Acknowledgments.}

We thank I. Agol and M. Culler for several helpful conversations. In particular, Agol has shown us a construction, using techniques from his paper [1], of surface bundles over the circle containing strongly irreducible splittings of high genus.

\section{References.}

[1] Ian Agol. Small 3-manifolds of large genus. Geom. Dedicata, 102:53-64, 2003. arXiv:math.GT/0205091.

[2] Jeffrey F. Brock. Weil-Petersson translation distance and volumes of mapping tori. Comm. Anal. Geom., 11(5):987-999, 2003. arXiv:math.GT/0109050.

[3] Jeffrey F. Brock, Richard D. Canary and Yair N. Minsky. The classification of Kleinian surface groups, II: The Ending Lamination Conjecture. aXiv:math.GT/0412006.

[4] Danny Calegari. Problems in foliations and laminations of 3-manifolds. In Topology and geometry of manifolds (Athens, GA, 2001), volume 71 of Proc. Sympos. Pure Math., pages 297-335. Amer. Math. Soc., Providence, RI, 2003. arXiv:math.GT/0209081. 
[5] Andrew J. Casson and Cameron McA. Gordon. Reducing Heegaard splittings. Topology Appl., 27(3):275-283, 1987.

[6] Daryl Cooper and Martin Scharlemann. The structure of a solvmanifold's Heegaard splittings. In Proceedings of 6th Gökova Geometry-Topology Conference, volume 23, pages 1-18, 1999. arXiv:math.GT/9803157.

[7] Marc Culler, Willam Jaco, and Hyam Rubinstein. Incompressible surfaces in once-punctured torus bundles. Proc. London Math. Soc. (3), 45(3):385-419, 1982.

[8] Willam Floyd and Alan Hatcher. Incompressible surfaces in puncturedtorus bundles. Topology Appl., 13(3):263-282, 1982.

[9] Rob Kirby. Problems in low-dimensional topology. Geometric topology (Athens, GA, 1993), 2:35-473, 1997. http://math.berkeley.edu/ kirby/problems.ps.gz.

[10] Tsuyoshi Kobayashi and Osamu Saeki. The RubinsteinScharlemann graphic of a 3-manifold as the discriminant set of a stable map. Pacific J. Math., 195(1):101-156, 2000. http://nyjm.albany.edu:8000/PacJ/2000/195-1-6nf.htm.

[11] Marc Lackenby. Heegaard splittings, the virtually Haken conjecture and property tau. arXiv:math.GT/0205327.

[12] D. D. Long and H. R. Morton. Hyperbolic 3-manifolds and surface automorphisms. Topology, 25(4):575-583, 1986.

[13] Howard A. Masur and Yair N. Minsky. Geometry of the complex of curves. I. Hyperbolicity. Invent. Math., 138(1):103-149, 1999. arXiv:math.GT/9804098.

[14] Yoav Moriah and Hyam Rubinstein. Heegaard structures of negatively curved 3-manifolds. Comm. Anal. Geom., 5(3):375-412, 1997.

[15] Dale Rolfsen. Knots and links. Publish or Perish Inc., Houston, TX, 1990. Corrected revision of the 1976 original.

[16] Hyam Rubinstein. Minimal surfaces in geometric 3-manifolds, to appear in Global theory of minimal surfaces, Proceedings of the Clay Institute workshop at MSRI. 
[17] Hyam Rubinstein and Martin Scharlemann. Comparing Heegaard splittings of non-Haken 3-manifolds. Topology, 35(4):1005-1026, 1996.

[18] Martin Scharlemann. Local detection of strongly irreducible Heegaard splittings. Topology Appl., 90(1-3):135-147, 1998.

[19] Martin Scharlemann. Heegaard splittings of compact 3-manifolds. In Handbook of geometric topology, pages 921-953. North-Holland, Amsterdam, 2002. arXiv:math.GT/0007144.

[20] Martin Scharlemann and Abigail Thompson. Heegaard splittings of (surface) $\times I$ are standard. Math. Ann., 295(3):549-564, 1993.

[21] Saul Schleimer. Strongly irreducible surface automorphisms. In Topology and geometry of manifolds (Athens, GA, 2001), volume 71 of Proc. Sympos. Pure Math., pages 287-296. Amer. Math. Soc., Providence, RI, 2003. arXiv:math.GT/0208110.

[22] Jennifer Schultens. The classification of Heegaard splittings for (compact orientable surface) $\times S^{1}$. Proc. London Math. Soc. (3), 67(2):425448, 1993.

[23] Abigail Thompson. The disjoint curve property and genus 2 manifolds. Topology Appl., 97(3):273-279, 1999.

[24] William P. Thurston. A norm for the homology of 3-manifolds. Mem. Amer. Math. Soc., 59(339):i-vi and 99-130, 1986.

[25] Friedhelm Waldhausen. Heegaard-Zerlegungen der 3-Sphäre. Topology, 7:195-203, 1968.

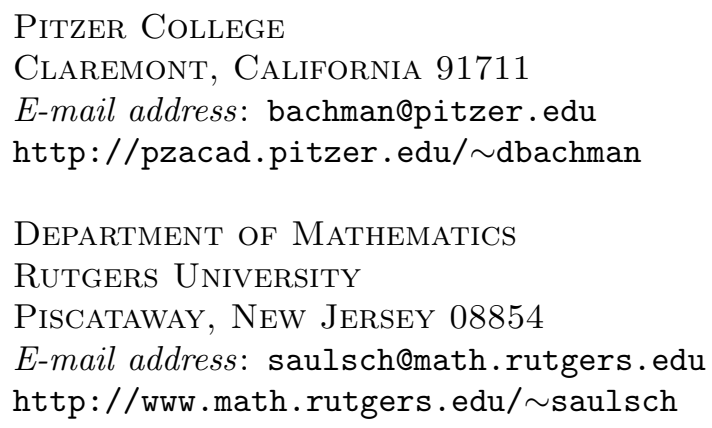

RECEIVED MAY 2, 2003. 